\title{
Apoio Social, Resiliência, Estresse de Minorias e Saúde Mental de Mulheres Lésbicas e Bissexuais
}

\author{
Fernanda de Oliveira Paveltchuk. \\ Juliane Callegaro Borsa ${ }^{2}$ \\ Bruno Figueiredo Damásio ${ }^{1}$ \\ ${ }^{1}$ Universidade Federal do Rio de Janeiro, Rio de Janeiro, Rio de Janeiro, Brasil \\ ${ }^{2}$ Pontifícia Universidade Católica do Rio de Janeiro (PUC-Rio),Rio de Janeiro, Rio de Janeiro, Brasil
}

\begin{abstract}
Resumo
Este estudo buscou avaliar os efeitos de moderação do apoio social e da resiliência na relação entre o Estresse de Minorias (EM) e desfechos de saúde mental. Participaram desse estudo 337 mulheres, sendo 42,43\% lésbicas $(n=143)$ e $57,57 \%$ bissexuais $(n=194)$, maiores de 18 anos $(M=26$ anos; $D P=17,3)$. As participantes responderam escalas de homofobia internalizada, vitimização, revelação da orientação sexual, felicidade subjetiva, satisfação com a vida, depressão, ansiedade e estresse, apoio social e resiliência. Foi realizada uma modelagem por equações estruturais para verificar o impacto dos estressores de minorias nas variáveis de bem-estar (BES) e psicopatologia (PSP), além de testar se apoio social e resiliência moderavam essas relações. Os resultados demonstraram que a resiliência moderou a relação entre vitimização e psicopatologia. Implicações clínicas são apontadas ao longo do artigo.

Palavras-chave: estresse de minorias, LGB, bem-estar, apoio social, resiliência
\end{abstract}

Social Support, Resilience, Minority Stress, and Mental Health Outcomes in Lesbian and Bisexual Women

\begin{abstract}
This study aimed to evaluate the moderating effects of social support and resilience in the relationship between Minority Stress (MS) and mental health outcomes. A total of 337 women over 18 years of age $(\mathrm{M}=26$ years, $\mathrm{SD}=17.3)$ participated in this study, including $42.43 \%$ lesbian, $(n=143)$ and $57.57 \%$ bisexual women $(n=194)$. The participants responded to scales that assessed internalized homophobia, victimization, disclosure of sexual orientation, subjective happiness, life satisfaction, depression, anxiety and stress, social support, and resilience. Structural equation modeling was performed to verify the impact of minority stressors on the well-being and psychopathology variables and to test whether social support and resilience moderated these associations. Results showed that resilience moderated the relationship between victimization and psychopathology. Clinical implications are discussed throughout the article.

Keywords: minority stress; social support; resilience; lgb; well-being
\end{abstract}

\section{Apoyo Social, Resiliencia, Estrés de Minorías y Salud Mental de Mujeres Lesbianas y Bisexuales}

\section{Resumen}

Este estudio buscó evaluar los efectos de moderación de apoyo social y resiliencia en la relación entre el Estrés de Mínorías e indicadores de salud mental. En este estudio participaron 337 mujeres, siendo 42,43\% lesbianas ( $\mathrm{n}=143$ ) y 57,57\% bisexuales ( $\mathrm{n}=194)$, mayores de 18 años de edad $(\mathrm{M}=26$ años, $\mathrm{DP}=17,3)$. Los participantes respondieron escalas de homofobia internalizada, victimización, revelación de orientación sexual, felicidad subjetiva, satisfacción con la vida, depresión, ansiedad y estrés, apoyo social y resiliencia. Se realizó el modelo de ecuaciones estructurales para verificar el impacto de los estresores de minorías en las variables de bienestar (BES) y psicopatología (PSP), además de testar si el apoyo social y la resiliencia moderaban estas relaciones. Los resultados demostraron que la resiliencia modera la relacción entre victimización y psicopatología. Implicaciones clínicas son señaladas a lo largo del artículo.

Palabras clave: estrés de minorías; lgb; bienestar; apoyo social; resiliencia

\section{Introdução}

Lésbicas, gays e bissexuais (LGB) apresentam maior comprometimento em indicadores de saúde mental quando comparados a seus pares heterossexuais (Pachankis, Hatzenbuehler, Rendina, Safren, \& Parsons, 2015). Entretanto, não há evidências científicas demonstrando que o maior risco a problemas psicopatológicos de pessoas LGB se deva a suas orientações sexuais em si (Pachankis et al., 2015). Ao contrário, maiores níveis de psicopatologia em indivíduos LGB são melhor explicados por condições psicossociais adversas enfrentadas por esses indivíduos. (Puckett, Horne, Herbitter, Maroney, \& Levitt, 2017). O estresse decorrente da homofobia, por exemplo, é parte da origem dessas dificuldades (Dunn, Gonzalez, Costa, Nardi, \& Iantaffi, 2013). A literatura vem destacando o conceito de minority stress (ou estresse de minorias, EM) como 
um modelo útil para compreender as condições internas e externas experienciadas por indivíduos LGB e de que maneira essas condições estão associadas à saúde mental desses indivíduos (Meyer, 2003; Pachankis et al., 2015). O modelo do EM abarca condições individuais e contextuais que, relacionadas entre si, podem explicar os maiores índices de psicopatologias em pessoas LGB.

O EM é adicional aos estressores comuns com os quais todas as pessoas têm contato. O diferencial é que pessoas estigmatizadas precisam se adaptar em maior nível às situações do que os não estigmatizados, por vivenciarem esses estressores específicos que se somam aos estressores cotidianos. O modelo do EM abarca três tipos de estressores: 1) experiências de vitimização (qualquer experiência de preconceito, rejeição, violência e agressão em relação à orientação sexual de uma pessoa); 2) HI (ideias aversivas acerca da própria homo ou bissexualidade); e 3) ocultação da identidade sexual (quando o indivíduo esconde de si e/ou de seus pares sua identidade LGB) (Meyer, 2003). Quanto ao estressor ocultação da identidade sexual, cabe a diferenciação entre os conceitos de outness e de disclosure: o conceito de outness diz respeito à revelação e à ocultação da orientação sexual, ou seja, o grau de abertura de uma pessoa LGB quanto a sua sexualidade, enquanto o conceito de disclosure se refere à revelação em si da orientação sexual (Meidlinger \& Hope, 2014).

A experiência do EM é diferente para cada grupo e, por isso, os modelos de EM devem levar a identidade sexual em consideração (Lewis, Kholodkov, \& Derlega, 2012). Diferenças de gênero e orientação sexual são cruciais, pois homens e mulheres terão contato com estressores de minoria específicos em graus diferentes, assim como gays, lésbicas e bissexuais terão vivências específicas que se refletirão em seus modelos de EM (Friedman \& Leaper, 2010; Szymanski, Kashubeck-West, \& Meyer, 2008). Além dos EM comuns, o grupo de bissexuais sofre ainda uma dificuldade adicional, o que leva à compreensão de que, por experienciarem dificuldades específicas e adicionais às de gays e lésbicas, bissexuais tenham um modelo próprio de estresse de minoria (Feinstein \& Dyar, 2017). A bissexualidade está associada a algumas desvantagens específicas no que concerne às interações sociais. Frequentemente, a identidade sexual desse grupo é taxada de "fase" ou de “indecisão" (Goodine, 2015; Kertzner, Meyer, \& Frost, 2009). Essa não legitimização da bissexualidade é denominada de "bifobia" e representa uma invisibilização dessa identidade (Herek, 2002; Kertzner et al., 2009). As desvantagens da população bissexual em relação às interações sociais e ao apoio social percebido são específicas, pois, além dos processos de estigmatização e discriminação derivadas do meio heterossexual, a população bissexual experiencia também esses processos na comunidade de gays e lésbicas (Kertzner et al., 2009). Como possíveis consequências dessa dupla experiência de preconceito sofrido estão maior dificuldade de obtenção e manutenção de rede de apoio social, maiores índices de confusão identitária em comparação a gays e lésbicas e menor revelação e expressão da identidade sexual (Balsam \& Mohr, 2007). Estudos têm demonstrado que o grupo de bissexuais apresenta maior prevalência de psicopatologias, como ansiedade e depressão, e maiores índices de ideação e tentativas de suicídio quando comparadas a gays, lésbicas e heterossexuais (Flanders, Dobinson, \& Logie, 2017; Taylor, 2018). Além disso, esse grupo tem experienciado os estressores específicos de minoria sexual, tal como a ocultação da orientação sexual, de forma diferente de gays e lésbicas. Uma vez que podem ser rejeitados por seus pares monossexuais, bissexuais que revelam sua orientação sexual apresentam níveis mais altos de abuso de substâncias e comprometimento na saúde mental em geral (Brewster \& Moradi, 2010; Feinstein, Dyar, Whitton, Newcomb, \& Mustanski, 2018).

O modelo teórico do EM propõe a existência de fatores de risco e proteção que podem auxiliar no enfrentamento do estresse vivenciado por indivíduos cuja identidade sexual é estigmatizada. Esses fatores influenciariam as respostas de gays, lésbicas e bissexuais ao preconceito (Dunn et al., 2013). Os fatores de proteção podem ser de nível individual, isto é, estarem relacionados a características dos próprios indivíduos, como estratégias de coping e autoaceitação, ou de nível grupal, como o apoio social (Meyer, 2003). Por exemplo, menores índices de apoio social estão associados a maior comprometimento na saúde mental de pessoas LGB (Bränström, 2017). O apoio dos pais, por sua vez, está associado a melhores indicadores de saúde, enquanto a rejeição familiar está ligada à depressão, ao abuso de substâncias e à ideação suicida (Zimmermann, Darnell, Rhew, Lee, \& Kaysen, 2015). A resiliência está relacionada ao EM como fator protetivo, especialmente em contexto comunitário, ou seja, como variável de grupo (Meyer, 2015). Supõe-se que indivíduos com estratégias de coping mais assertivas e maior apoio social apresentem maiores níveis de resiliência, repercutindo, portanto, em menores níveis de HI e maiores graus de autoaceitação, o que permitiria um maior conforto com a identidade sexual, acarretando em maior qualidade de vida (Meyer, 2003). 
O presente estudo buscou verificar os níveis de EM e psicopatologia em mulheres lésbicas e bissexuais no Brasil e observar se a resiliência e o apoio social poderiam moderar essas relações.

\section{Método}

\section{Participantes}

Participaram deste estudo 337 mulheres, sendo $42,43 \%$ lésbicas $(n=143)$ e $57,57 \%$ bissexuais $(n=$ 194), assumidas $(67,96 \% ; n=227)$ ou não $(32,03 \% ; n$ =107), maiores de 18 anos de idade $(M=26$ anos; $D P$ $=17,3)$. A renda familiar declarada pelas participantes variou de três a 10 salários mínimos $(60,1 \% ; n=201)$. A maioria das respondentes cursava ou já havia concluído o ensino superior $(78,2 \% ; n=261)$. Não houve cálculo amostral para definição do número de participantes. Foram incluídas no estudo todas as mulheres que afirmaram ser lésbicas ou bissexuais.

\section{Instrumentos}

Questionário sociodemográfico. Esse instrumento visa a levantar informações sociodemográficas, com aspectos referentes ao local de residência, gênero, instituição de ensino, idade, estado civil, constituição familiar, religião, escolaridade, vínculo empregatício, renda etc.

Escala de Satisfação com a Vida (Diener et al., 1985, adaptada por Gouveia, Milfont, Fonseca, \& Coelho, 2009). A Escala de Satisfação com a Vida avalia a satisfação do indivíduo a respeito de sua própria vida. Possui cinco itens, que são respondidos em escala Likert de sete pontos, com variação de 1 (discordo totalmente) a 5 (concordo totalmente). No presente estudo, a fidedignidade da escala foi $\alpha=0,89$.

Escala de Felicidade Subjetiva (Damásio, Zanon, \& Koller, 2014). A Escala de Felicidade Subjetiva (EFS) avalia, a partir da percepção das próprias pessoas, seus níveis de felicidade (componente emocional do bem-estar subjetivo). O instrumento é composto por quatro itens, respondidos em uma escala de sete pontos. No presente estudo, a fidedignidade da escala foi $\alpha=0,89$.

Escala de Resiliência - Versão 14 itens (Wagnild, 2010, validado por Damásio, Borsa, \& da Silva, 2011). O instrumento, composto por 14 itens, investiga a capacidade do indivíduo em solucionar e superar dificuldades e adversidades, com pontuação de escala Likert variando de 1 (discordo totalmente) a 7 (concordo totalmente). No presente estudo, a fidedignidade da escala foi $\alpha=0,90$.

Escala de Apoio Social do Estudo Pró-Saúde (Griep et al., 2005). O instrumento consiste em 19 itens, que avaliam a frequência com que o participante considera ter com quem contar para lazer, informações, afeto e ajuda, com pontuação de 1 (nunca) a 5 (sempre). Além disso, há duas perguntas abertas sobre a quantidade de amigos e familiares com quem o respondente pode falar sobre quase tudo. Essas perguntas não foram utilizadas no presente estudo. Neste, a fidedignidade da escala foi $\alpha=0,96$.

Escala de Depressão, Ansiedade e Stress (DASS - 21; Apóstolo, Mendes, \& Azeredo, 2006). O instrumento consiste em 21 itens que investigam indicadores de depressão, ansiedade e estresse, relacionando-a com a qualidade de vida e a personalidade. As respostas podem variar de 0 (Não se aplicou a mim de forma nenhuma) a 3 (Aplicou-se muito a mim ou na maior parte do tempo). Neste estudo, a fidedignidade dos fatores foi: depressão, $\alpha=$ 0,94; ansiedade $\alpha=0,89$; estresse $\alpha=0,91$.

Experiências com Vitimização (Dunn et al, 2013). O instrumento consiste em sete itens referentes a experiências de violência física ou verbal decorrentes da homofobia. O indivíduo marca na opção que melhor descreva com que frequência uma situação acontece ou tenha acontecido em sua vida. No presente estudo, a fidedignidade da escala foi $\alpha=0,68$.

Sentimentos sobre Homossexualidade/Bissexualidade (Dunn et al, 2013). O instrumento consiste em sete itens a respeito dos sentimentos de um indivíduo acerca de sua orientação sexual homossexual ou bissexual. A pontuação é feita de 1 (discordo totalmente) a 7 (concordo totalmente), no modelo de escala Likert. No presente estudo, a fidedignidade da escala foi $\alpha=0,65$.

"Sair do Armário": Assumindo a Sexualidade (Dunn et al, 2013). O instrumento consiste em quatro itens: família, amigos heterossexuais, amigos LGBT e colegas de trabalho. $\mathrm{O}$ indivíduo deve informar para quantas pessoas assumiu sua orientação sexual (ninguém, poucos, muitos, todos ou se o item não se aplica). No presente estudo, a fidedignidade da escala foi $\alpha=0,67$.

\section{Procedimentos de Coleta de Dados}

A coleta de dados foi realizada por meio de uma plataforma virtual e o link para a participação na pesquisa foi divulgado em redes sociais e por e-mail. Além disso, foi utilizada a técnica bola de neve, em que participantes do estudo indicam outros possíveis interessados na pesquisa (Patton, 1990).

\section{Procedimentos Éticos}

Este estudo foi conduzido após aprovação pelo Comitê de Ética em Pesquisa do (do Centro de Filosofia 
e Ciências Humanas (CFCH) da Universidade Federal do Rio de Janeiro) (CAEE: 40922815.5.0000.5582). Todas as suas etapas foram realizadas de maneira ética e metodologicamente adequada, de acordo com as Diretrizes e Normas Regulamentadoras de Pesquisa envolvendo Seres Humanos (Resolução n. 466/12 do Conselho Nacional de Saúde).

\section{Procedimentos de Análise de Dados}

Inicialmente, foram realizadas correlações de Pearson com o objetivo de investigar as relações entre todas as variáveis do estudo, a saber: Saída do Armário (SARM), Experiências de Vitimização (VIT) e Homofobia Internalizada (HI), Felicidade Subjetiva (FS), Satisfação com a Vida (SV), Resiliência (RES), Apoio Social (AS), Depressão (DEP), Ansiedade (ANS) e Estresse (EST).

Após investigar as relações entre as variáveis, buscou-se analisar, por meio de uma path analysis, subtipo de modelagem por equações estruturais de multimoderação, se os recursos psicossociais RES e AS poderiam moderar o impacto do EM nos indicadores de bem-estar subjetivo e psicopatologia. Os procedimentos de modelagem por equações estruturais foram realizados utilizando o método de extração Weighted Least Squares Mean and Variance-Adjusted (WLSMV). Foi utilizado o método de estimação WLSMV, por este ser um método de extração adequado para dados ordinais (Muthén \& Muthén, 2010). A adequação de ajuste dos modelos foi avaliada por meio dos seguintes índices: CFI, TLI, e RMSEA. De acordo com as diretrizes utilizadas (Brown, 2006), os valores de CFI e TLI devem estar acima de 0,90 . Valores de RMSEA devem estar abaixo de 0,08 , sendo que o limite superior do intervalo de confiança $(90 \%)$ não deve ser superior a 0,10 . As análises foram realizadas utilizando o software Mplus v. 7.11 (Muthén \& Muthén, 2010).

\section{Resultados}

Inicialmente buscou-se avaliar as relações entre as variáveis do EM (saída do armário (SARM), experiências de vitimização (VIT) e homofobia internalizada (HI), com os índices de felicidade subjetiva (FS), satisfação com a vida (SV), resiliência (RES), apoio social (AS), depressão (DEP), ansiedade (ANS) e estresse (DAS). Conforme pode ser visto, a variável SARM tem correlação negativa significativa $(\phi<0,01)$ com SV, FS, RES e AS. Quanto às variáveis ANS, DEP e DAS, sua correlação é positiva e significativa $(p<0,05)$. A variável
VIT se correlaciona significativamente de forma negativa com SV e FS ( $p<0,01$ e $p<0,05$, respectivamente) e positiva significativa $(\phi<0,01)$ com DEP, ANS e DAS. Em relação à variável HI, há correlações positivas significativas $(p<0,01)$ com DEP e DAS e negativas significativas $(p<0,01)$ com SV, FS, RES e AS. Não houve correlações significativas entre ANS e HI, RES e VIT, e AS e VIT (ver Tabela 1).

Com o objetivo de analisar com maior profundidade as relações entre as variáveis, foi realizada uma modelagem por equações estruturais utilizando todos os indicadores acima descritos. A fim de tornar o estudo mais parcimonioso, foram criadas duas variáveis latentes de segunda ordem, a saber, bem-estar subjetivo (BES; composto pelas variáveis latentes felicidade subjetiva e satisfação com a vida) e Psicopatologia (PSP; composto pelas variáveis latentes de estresse, depressão e ansiedade). Os índices de ajuste suportaram a estrutura de segunda ordem para ambos os indicadores BBES, $\chi^{2}=61.562 ; d f=25$; RMSEA $(90 \%$ IC) $=0.066$ $(0,046-0,087)$; CFI $=0,976$; TLI $=0,966]$; PSP, $\chi^{2}=$ 408,367; $d f=186$; RMSEA $(90 \%$ IC) $=0,060(0,052-$ 0,068); CFI $=0,986$; TLI $=0,985$ (Tabelas 2 e 3 ).

Posteriormente, foi implementada uma modelagem por equações estruturais, visando a avaliar como os indicadores do EM impactavam nos índices de BES e PSP. Inseriu-se como variáveis moderadoras os índices de AS e RES. Os índices de ajuste da modelagem por equações estruturais com multimoderação foram: $\chi^{2}=1616.461 ; d f=1064 ; \operatorname{RMSEA}(90 \%$ IC) $=0,039$ $(0,036-0,043)$; CFI $=0,975$; TLI $=0,974]$. Os resultados do modelo são apresentados na Figura 1.

Tabela 1.

Correlação entre as Variáveis

\begin{tabular}{llll}
\hline & VIT & HI & SARM \\
\hline $\begin{array}{llll}\text { Satisfação com a } \\
\text { vida }\end{array}$ & $-0,141^{* *}$ & $-0,288^{* *}$ & $-0,191^{* *}$ \\
Felicidade subjetiva & $-0,116^{*}$ & $-0,272^{* *}$ & $-0,180^{* *}$ \\
Depressão & $0,147^{* *}$ & $0,169^{* *}$ & $0,111^{*}$ \\
Ansiedade & $0,176^{* *}$ & 0,081 & $0,117^{*}$ \\
Estresse & $0,177^{* *}$ & $0,144^{* *}$ & $0,108^{*}$ \\
Resiliência & $-0,047$ & $-0,189^{* *}$ & $-0,170^{* *}$ \\
Apoio social & $-0,074$ & $-0,368^{* *}$ & $-0,238^{* *}$ \\
\hline
\end{tabular}

Nota. VIT = Experiências de vitimização; $\mathrm{HI}=$ Homofobia internalizada; SARM = Saída do armário. ${ }^{*} p<0,05,{ }^{*} p<0,01$.

Psico-USF, Bragança Paulista, v. 25, n. 3, p. 403-414, jul./set. 2020 
Tabela 2.

Cargas Fatoriais da Estrutura de Segunda Ordem BES

\begin{tabular}{lcccc}
\hline Indicadores de BES & Indicador & Carga fatorial & Erro padrão & valor- $p$ \\
\hline Satisfação com a vida & Item 1 & 0.822 & 0.024 & 0.000 \\
& Item 2 & 0.761 & 0.030 & 0.000 \\
& Item 3 & 0.912 & 0.015 & 0.000 \\
& Item 4 & 0.779 & 0.026 & 0.000 \\
Felicidade subjetiva & Item 5 & 0,710 & 0,031 & 0,000 \\
& Item 1 & 0,909 & 0,018 & 0,000 \\
& Item 2 & 0,875 & 0,019 & 0,000 \\
& Item 3 & 0,786 & 0,028 & 0,000 \\
& Item 4 & $-0,680$ & 0,041 & 0,000 \\
BES (Modelo de segunda-ordem) & Satisfação com a vida & 0,833 & 0,074 & 0,000 \\
& Felicidade Subjetiva & 0,967 & 0,066 & 0,000 \\
\hline
\end{tabular}

Tabela 3.

Cargas Fatoriais da Estrutura de Segunda Ordem PSP

\begin{tabular}{|c|c|c|c|c|}
\hline $\begin{array}{l}\text { Indicadores de } \\
\text { Psicopatologia }\end{array}$ & Indicador & Carga fatorial & Erro padrão & valor- $p$ \\
\hline \multirow[t]{7}{*}{ Depressão } & Item 1 & 0,904 & 0,014 & 0,000 \\
\hline & Item 2 & 0,801 & 0,024 & 0,000 \\
\hline & Item 3 & 0,927 & 0,011 & 0,000 \\
\hline & Item 4 & 0,928 & 0,011 & 0,000 \\
\hline & Item 5 & 0,871 & 0,017 & 0,000 \\
\hline & Item 6 & 0,856 & 0,018 & 0,000 \\
\hline & Item 7 & 0,816 & 0,021 & 0,000 \\
\hline \multirow[t]{7}{*}{ Ansiedade } & Item 1 & 0,586 & 0,043 & 0,000 \\
\hline & Item 2 & 0,735 & 0,029 & 0,000 \\
\hline & Item 3 & 0,796 & 0,028 & 0,000 \\
\hline & Item 4 & 0,861 & 0,021 & 0,000 \\
\hline & Item 5 & 0,886 & 0,020 & 0,000 \\
\hline & Item 6 & 0,749 & 0,028 & 0,000 \\
\hline & Item 7 & 0,813 & 0,025 & 0,000 \\
\hline \multirow[t]{7}{*}{ Estresse } & Item 1 & 0,796 & 0,026 & 0,000 \\
\hline & Item 2 & 0,798 & 0,024 & 0,000 \\
\hline & Item 3 & 0,887 & 0,016 & 0,000 \\
\hline & Item 4 & 0,816 & 0,022 & 0,000 \\
\hline & Item 5 & 0,891 & 0,016 & 0,000 \\
\hline & Item 6 & 0,757 & 0,027 & 0,000 \\
\hline & Item 7 & 0,831 & 0,022 & 0,000 \\
\hline \multirow{3}{*}{$\begin{array}{l}\text { PSP (Modelo de } \\
\text { segunda ordem) }\end{array}$} & Depressão & 0,877 & 0,018 & 0,000 \\
\hline & Ansiedade & 0,951 & 0,015 & 0,000 \\
\hline & Estresse & 0,943 & 0,012 & 0,000 \\
\hline
\end{tabular}


Conforme pode ser visto, houve impacto significativo das variáveis do EM em desfechos de BES e PSP. As cargas baixas ou médias são parte do esperado, pois a orientação sexual é apenas uma das facetas da realidade subjetiva da pessoa LGB. A variável SARM impactou de forma negativa significativa os índices de PSP, mas não alterou significativamente o BES. A variável VIT impactou significativamente de forma negativa o BES e positivamente o agrupamento PSP. A variável $\mathrm{HI}$ impactou de forma significativa negativa a variável BES, mas não teve impacto na variável PSP. Em relação às análises de moderação, apenas um efeito significativo foi encontrado: a resiliência moderou as relações entre experiências de vitimização e psicopatologia $(\beta=0,04 ; p<0,05)$. Os escores de resiliência foram divididos em três grupos (1 desvio padrão abaixo da média; \pm 1 desvio padrão da média e 1 desvio padrão acima da média). Os resultados demonstraram que, para pessoas com baixo nível de resiliência, não houve impacto significativo da vitimização nos índices de psicopatologia $(\beta=0,312 ; p=$ $0,366)$. Para pessoas com médio e alto nível de resiliência, o impacto foi significativo (médio, $\beta=0,890$; $p<0,001$; alto, $\beta=1,468 ; p<0,001$ ). Quanto maior o nível de resiliência, maior foi o impacto da vitimização nos índices de psicopatologia (Ver Figura 2).

\section{Discussão}

No presente estudo, buscou-se investigar como as variáveis de EM poderiam impactar os níveis de bem-estar subjetivo e psicopatologia em uma amostra brasileira de mulheres lésbicas e bissexuais. Os resultados da modelagem por equações estruturais demonstraram não haver impacto significativo da saída do armário no bem-estar subjetivo. No entanto, houve

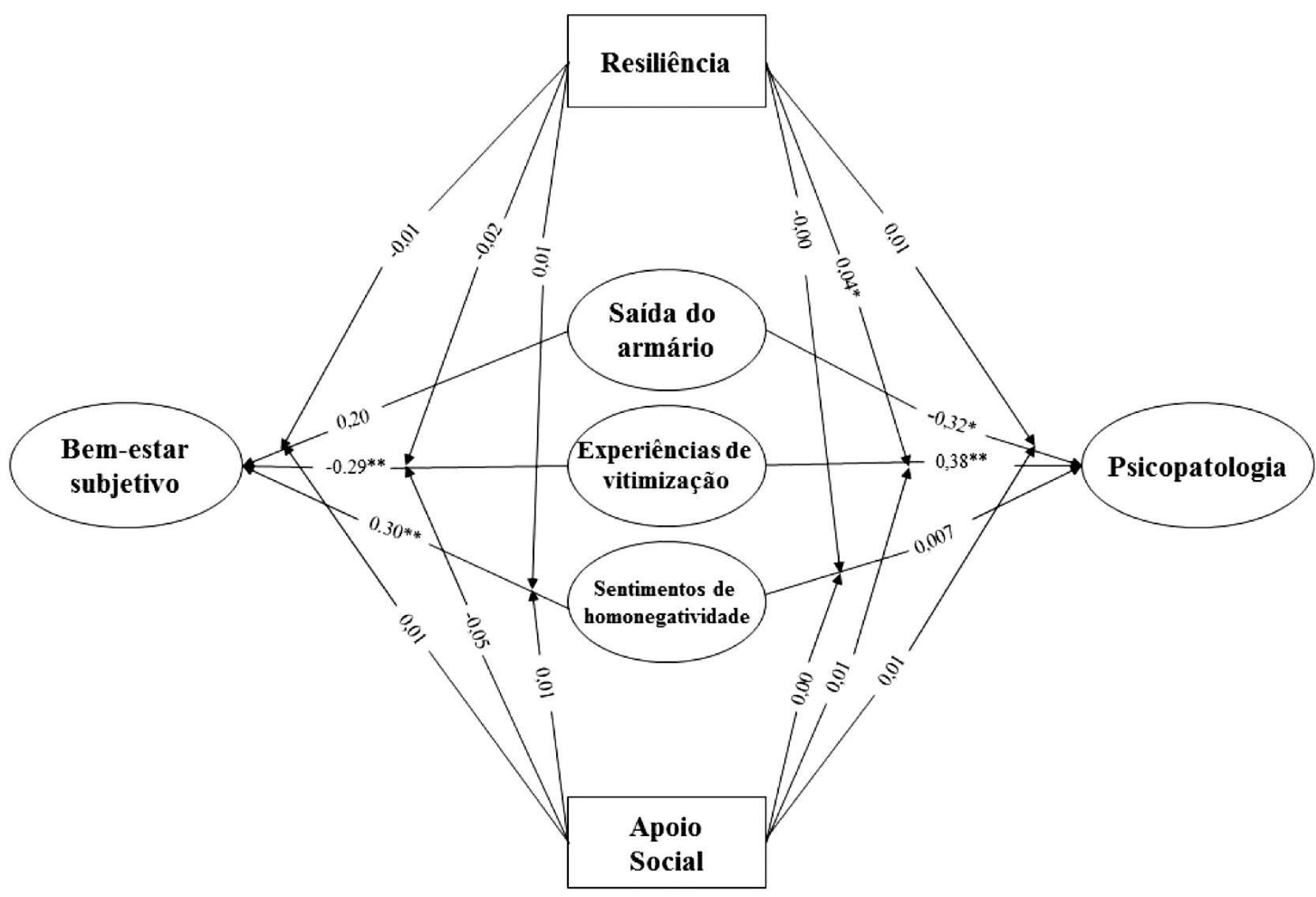

Nota. ${ }^{*} p<0,05 ; * * p<0,01$.

A figura é um gráfico representacional. Os itens que compõem cada dimensão não são apresentados por motivos de parcimônia.

Figura 1. Modelo de equações estruturais das relações entre EM, BES e PSP moderados por RES e AS. 


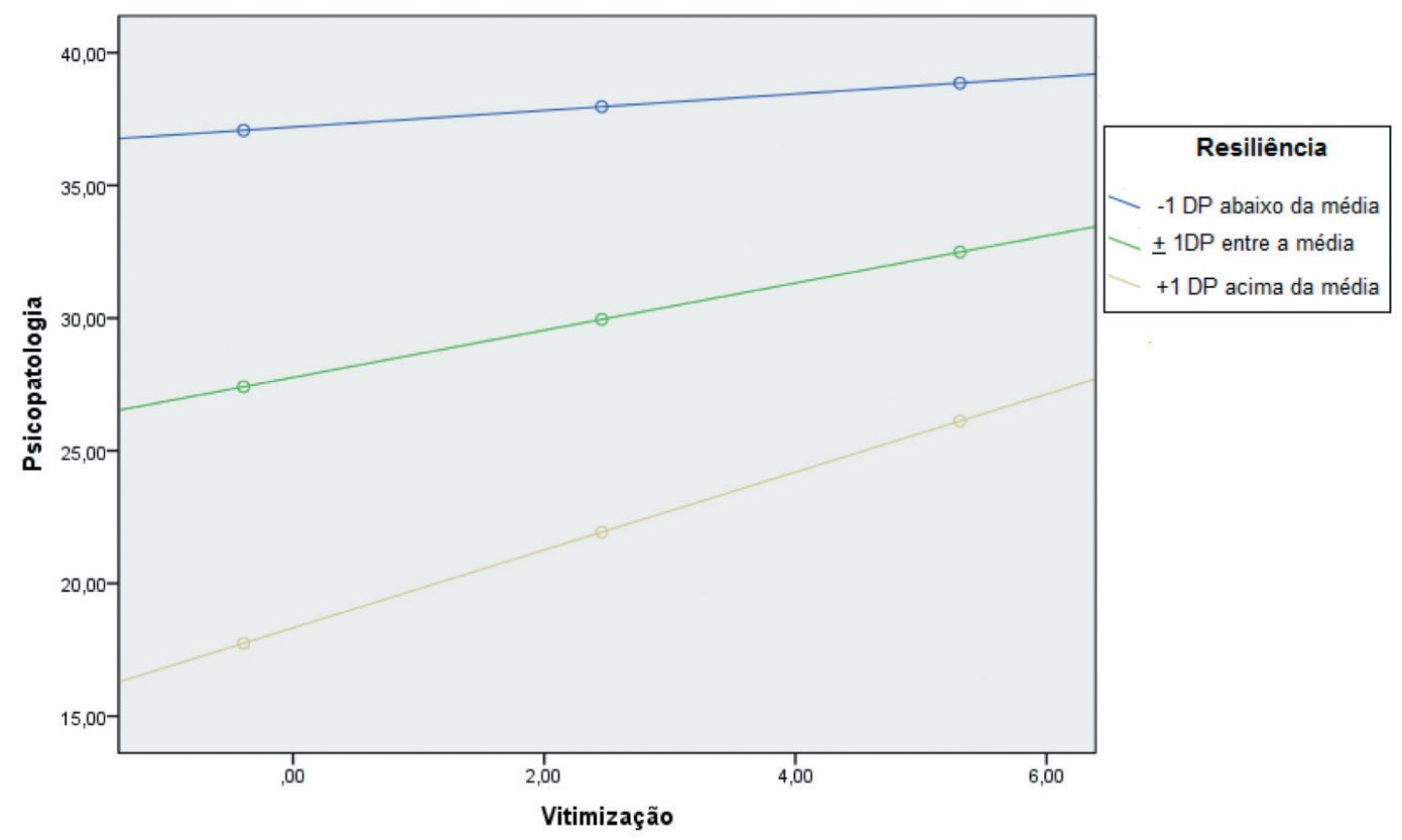

Figura 2. Gráfico da análise de moderação entre resiliência, vitimização e psicopatologia.

relação negativa significativa entre a saída do armário e os índices de psicopatologia. Os resultados estão parcialmente de acordo com a literatura, considerando que a revelação da identidade sexual tem sido um fator significativo no bem-estar subjetivo e psicológico de respondentes de alguns estudos, e não de outros (Balsam, Martell, \& Martell, 2006; Martell, Safren, \& Prince, 2004). Cada experiência de saída do armário é singular e, por isso, deve-se levar em conta outras variáveis, como diferenças a nível de personalidade e familiar ou social de cada indivíduo (Drescher, 2014). É possível que, dependendo de características individuais e do teor de aceitação do meio, os efeitos no bem-estar e na saúde mental possam ou não ser relevantes. Sair do armário pode diminuir a sensação de ser diferente por permitir à pessoa LGB encontrar um grupo de referência; desse modo, as crenças de inadequação ou defeituosidade podem ser atenuadas. Porém, essa mudança nem sempre acontece (Martell, Safren, \& Prince, 2004). Além disso, revelar a identidade sexual é um processo contínuo já que, ao longo da vida, uma mulher lésbica ou bissexual pode repetir a mesma ação muitas vezes, para pessoas distintas (Drescher, 2014). Os desfechos psicológicos desse fato podem estar associados a mais fatores, como capacidades de enfrentamento individuais e fatores de risco e proteção específicos. Sair do armário também pode significar estar exposto a um número maior de riscos, já que ser uma pessoa LGB pode ser perigoso, nos padrões da cultura vigente (Martell, Safren, \& Prince, 2004). Por outro lado, a saída do armário pode estar associada a menores índices de psicopatologia, como no presente estudo, uma vez que, de acordo com o modelo do EM (Meyer, 2003), a ocultação da identidade sexual funcionaria como um estressor. Nesse sentido, revelar a identidade LGB poderia auxiliar num processo de autoaceitação e evitar o desenvolvimento de psicopatologias por tirar o indivíduo do estado de alerta derivado da ocultação da sexualidade (Drescher, 2014).

Em relação aos sentimentos de HI, por sua vez, é importante considerar que o estigma relacionado às identidades LGB pode se transformar em ódio a si mesmo (Borrillo, 2010). Características sociais e do meio são fatores importantes no desenvolvimento de filtros desadaptativos de interpretação das situações, esquemas desadaptativos ou crenças disfuncionais (Beck, 1997; Young, Klosko, \& Weishaar, 2008). Mulheres lésbicas e bissexuais são alvo de processos de estigmatização e discriminação, podendo gerar ou fortalecer essas crenças a respeito de sua própria sexualidade (i.e., HI) (Martell, Safren, \& Prince, 2004). No presente estudo, o construto $\mathrm{HI}$ impactou significativamente o bem-estar subjetivo, mas sua relação com os índices de 
psicopatologia das respondentes não foi significativa. Compreende-se que sentimentos de HI possam atingir inicialmente o bem-estar subjetivo da população LGB antes de alcançar o bem-estar psicológico. Uma vez que a Organização Mundial de Saúde (OMS, 1948) define saúde como não apenas a ausência de patologias, mas como um estado de bem-estar, hipotetiza-se que esse impacto está relacionado a um nível gradativo de impacto, de modo que o bem-estar seria atingido antes de impactar em psicopatologias mais severas. Assim, cabe destacar que parte da felicidade subjetiva e da satisfação com a vida das mulheres lésbicas e bissexuais que participaram deste estudo é significativamente comprometida por sentimentos de $\mathrm{HI}$ a respeito de si mesmas.

As mulheres lésbicas e bissexuais acumulam dois tipos de discriminação: a violência de gênero e a homofobia (Borrillo, 2010). A homofobia é a atitude de hostilidade contra a população LGB (Borrillo, 2010) que explica em grande parte as experiências de vitimização vivenciada por esses indivíduos. As experiências de vitimização (ou seja, agressões físicas e verbais sofridas) podem impactar fortemente as crenças e a subjetividade de pessoas não heterossexuais (Borrillo, 2010; Meyer, 2003). No presente estudo, as experiências de vitimização mostraram ter relação positiva significativa com a psicopatologia, e relação negativa significativa no que diz respeito ao bem-estar subjetivo, sugerindo que quanto mais experiências de preconceito vivenciadas, menores são os índices de BES e maiores os índices de PSP das participantes. As experiências de vitimização podem impactar tanto o bem-estar subjetivo quanto o psicológico, pois é a partir do ambiente hostil que as crenças e sentimentos a respeito da própria sexualidade, assim como o ritual de revelação da identidade sexual, fazem sentido (Pachankis et al., 2015). Além disso, a constante ameaça de atos de discriminação pode manter as mulheres lésbicas e bissexuais em estado de alerta e aumentar as sensações de insegurança, ansiedade e medo, afetando a qualidade de vida de mulheres não heterossexuais (Levounis, Drescher, \& Barber, 2014). Esse constante estado de alerta pode levar ao estresse crônico, podendo impactar a saúde física e mental das respondentes (Pachankis et al., 2015).

Nos testes de moderação, as variáveis apoio social e resiliência não se apresentaram como mediadoras das relações entre as variáveis do EM e desfechos de BES e PSP, exceto pela moderação significativa da resiliência na relação entre experiências de vitimização e psicopatologia. Em relação à ausência de moderação do apoio social entre o EM e os desfechos de BES e PSP, duas hipóteses são apresentadas: a primeira diz respeito às características da amostra. Como a pesquisa foi divulgada por meio de links e grupos e coletivos LGBT, é possível que o engajamento da amostra ao movimento LGBT justifique os altos índices de apoio social (AS) reportados.

Como não houve grande variabilidade nos índices de AS, não foi possível gerar uma análise adequada da moderação (a amplitude da escala de apoio social varia de 19 a 95 e, para essa amostra, $M=76,21 ; D P=$ 14,83). Além disso, a escala utilizada para medir o apoio social pode ter se revelado uma limitação do estudo por sua escala de respostas ser em frequência, sem caracterizar a qualidade e o tipo de AS recebido (famíliar, de amigos, colegas de trabalho etc.), pois uma pessoa pode reportar apoio social com frequência de média a alta mesmo sem obter a aceitação da família e dos pares em relação a sua orientação sexual, especificamente. $\mathrm{Ou}$ seja, a não investigação da fonte de apoio social pode ter impactado os resultados do presente estudo. Outra possibilidade é que alguém que seja aceito por poucas pessoas tenha pontuações tão altas quanto quem tem aceitação de grande número de família e amigos, já que a frequência não necessariamente resulta em qualidade desse apoio. Ademais, é possível que os níveis de AS reportados tenham sido médios ou altos por se trater de uma escala de AS geral e não especificamente relacionado à orientação sexual das respondentes. Uma mulher lésbica ou bissexual poderia apresentar índices médios ou altos de AS geral, mas baixos de AS especificamente relacionados à sua sexualidade (ela poderia receber apoio material ou emocional em geral, mas não poder, por exemplo, receber conselhos a respeito de seus relacionamentos românticos com outras mulheres). Futuros estudos serão conduzidos para testar as hipóteses aqui apresentadas.

Quanto à resiliência, esse resultado também não era esperado, haja vista que a resiliência, assim como o apoio social, deveria atuar como um fator protetivo, conforme apresenta a literatura (Dunn et al., 2013; Pachankis et al., 2015; Zimmermann, Darnell, Rhew, Lee e Kaysen, 2015). É importante perceber, porém, que pessoas com alto nível de resiliência apresentam escores menores em psicopatologia $(r=-0,53, p<0,01)$, mas índices semelhantes de vitimização, com pessoas com menores índices de resiliência $(r=0,039, p=0,47$, n.sig). Assim, é possível hipotetizar que a vitimização em pessoas com alto nível de resiliência possa impactar mais fortemente os indicadores de psicopatologia desse grupo, quando comparado com aqueles outros grupos 
que já tem níveis de psicopatologia altos (i.e., grupos que tem escores menores em resiliência). A ausência de relações de moderação também poderia ser explicada pelo envolvimento em movimentos sociais por parte das respondentes, já que estas foram acessadas em grupos de temática LGB e de combate à homofobia e ao sexismo em redes sociais, e estarem associadas aos movimentos feminista e LGBT, podendo já ter obtido apoio social dos companheiros de movimentos sociais e desenvolvido resiliência a partir da vivência de aceitação de suas identidades sexuais, solidificada pela participação e engajamento nos grupos de militância LGBT. Vale destacar, além disso, que a resiliência é um construto contextual, mais do que individual e que, por isso, medi-la por instrumentos escalares possivelmente não traz à luz resultados mais próximos da realidade (Reppold, Mayer, Almeida, \& Hutz, 2012).

É importante salientar, porém, que essas hipóteses não são conclusivas, merecendo a execução de futuros estudos. Neste estudo, a maioria das respondentes afirmou ser branca, possuir renda familiar entre 3 e 10 salários mínimos e ensino superior incompleto. Pelo fato de a amostra ser bastante homogênea nesse sentido, não houve uma ampla diversidade que seja mais próxima às várias realidades brasileiras.

\section{Considerações Finais}

Pertencer a um grupo estigmatizado pode trazer impactos clínicos para o bem-estar subjetivo e a saúde mental (Pachankis et al., 2015). A população LGB possui estressores específicos, ligados aos processos de estigmatização e discriminação, que não estão presentes na vida de seus pares heterossexuais (Meyer, 2003). Mulheres lésbicas e bissexuais sofrem ainda, além das experiências de preconceito, a opressão de gênero (Borrillo, 2010). Nesse contexto, o presente estudo teve por objetivo compreender as relações entre as variáveis do estresse de minorias e os índices de bem-estar subjetivo e de psicopatologia da amostra de mulheres lésbicas e bissexuais respondente. Além disso, também buscou-se avaliar a moderação da resiliência e do apoio social em cada uma das relações supracitadas. Houve impacto significativo da variável saída do armário no desenvolvimento de psicopatologias, assim como da homofobia internalizada no bem-estar subjetivo das participantes. Além disso, houve impacto das experiências de vitimização tanto no quadro de bem-estar subjetivo quanto no de psicopatologia. A resiliência se mostrou moderadora das experiências de vitimização que impactavam os níveis de psicopatologia do grupo de mulheres respondente. Os dados coletados são úteis no sentido de consistirem em uma análise mais ampla do contexto de saúde mental e recursos de enfrentamento e fatores de proteção ou risco das mulheres lésbicas e bissexuais do país. Porém, cabe destacar as limitações do estudo: ao utilizar da técnica da bola de neve (Patton, 1990) para coleta on-line, a amostra terminou por se revelar bastante homogênea, tornando os dados expressivos para o grupo acessado e semelhantes com características sociodemográficas similares, não atingindo a diversidade de realidades de mulheres lésbicas e bissexuais no Brasil. Recomenda-se que novas pesquisas na temática sejam feitas, com maior número de participantes, para que os resultados possam indicar com mais precisão a realidade nacional desse grupo.

\section{Referências}

Apóstolo, J. L. A., Mendes, A. C., \& Azeredo, Z. A. (2006). Adaptação para a língua portuguesa da Depression, Anxiety and Stress Scale (DASS). Revista Latino-americana de Enfermagem, 14(6). doi: 10.1590/S0104-11692006000600006

Balsam, K. F., \& Mohr, J. J. (2007). Adaptation to sexual orientation stigma: A comparison of bisexual and lesbian/gay adults. Journal of Counseling Psychology, 54(3), 306-319. doi: 10.1037/0022-0167.54.3.306

Balsam, K. F., Martell, C. R., \& Safren, S. A. (2006). Lesbian, gay, and bisexual affirmative cognitivebehavioral therapy. Em P. Hays \& G. Iwamasa (Eds.). Culturally responsive cognitive-behavioral therapy: Assessment, practice, and supervision, (pp. 223-243). Washington DC, EUA: American Psychological Association Press.

Beck, J. (1997). Terapia cognitiva: Teoria e prática. Porto Alegre, Brasil: Artmed.

Borrillo, D. (2010). Homofobia: História e crítica de um preconceito. Belo Horizonte, Brasil: Autêntica Editora.

Bränström, R. (2017). Minority stress factors as mediators of sexual orientation disparities in mental health treatment: a longitudinal population-based study. Journal of Epidemiology and Community Health, 2016. doi: 10.1136/jech-2016-207943

Brewster, M. E., \& Moradi, B. (2010). Perceived experiences of anti-bisexual prejudice: Instrument 
development and evaluation. Journal of counseling psychology, 57(4), 451-468. Doi: 10.1037/a0021116

Brown, T. A. (2006). Confirmatory factor analysis for applied research. Nova York, EUA: The Guilford Press.

Damásio, B. F., Borsa, J. C., \& da Silva, J. P. (2011). 14 -Item Resilience Scale (RS-14): Psychometric properties of the Brazilian version. Journal of Nursing Measurement, 19(3), 131-145. doi: 10.1891/1061-3749.19.3.131

Damásio, B. F., Zanon, C., \& Koller, S. H. (2014). Validation and psychometric properties of the Brazilian version of the Subjective Happiness Scale. Universitas Psychologica, 13(1), 1-13. doi: 10.11144/Javeriana. UPSY13-1.vppb

Diener, E. D., Emmons, R. A., Larsen, R. J., \& Griffin, S. (1985). The satisfaction with life scale. Journal of personality assessment, 49(1), 71-75. Doi: 10.1207/ s15327752jpa4901_13

Drescher, J. (2014). O que tem em seu armário? Em P. Levounis, J. Drescher \& M. E. Barber, (Eds.) (2014). O livro de casos clínicos GLBT, (pp. 21-35). Porto Alegre, Brasil: Artmed.

Dunn, T. L., Gonzalez, C. A., Costa, A. B., Nardi, H. C., \& Iantaffi, A. (2014). Does the minority stress model generalize to a non-us sample? An examination of minority stress and resilience on depressive symptomatology among sexual minority men in two urban areas of Brazil. Psychology of Sexual Orientation and Gender Diversity, 1(2), 117-131. doi: $10.1037 / \operatorname{sgd} 0000032$

Feinstein, B. A., \& Dyar, C. (2017). Bisexuality, minority stress, and health. Current sexual health reports, 9(1), 42-49. doi: 10.1007/s11930-017-0096-3

Feinstein, B. A., Dyar, C., Li, D. H., Whitton, S. W., Newcomb, M. E., \& Mustanski, B. (2018). The longitudinal associations between outness and health outcomes among gay/lesbian versus bisexual Emerging Adults. Archives of sexual behavior, 1-16. doi: 10.1007/s10508-018-1221-8

Flanders, C. E., Dobinson, C., \& Logie, C. (2017). Young bisexual women's perspectives on the relationship between bisexual stigma, mental health, and sexual health: A qualitative study. Critical Public Health, 27(1), 75-85. doi: 10.1080/09581596.2016.1158786

Friedman, C., \& Leaper, C. (2010). Sexual-minority college women's experiences with discrimination:
Relations with identity and collective action. Psychology of Women Quarterly, 34, 152-164. doi:10.1111/j.1471-6402.2010.01558.x

Goodine, R. (2015). The Silent B: The Erasure of Bisexuality. In: O’Neill, B., Swan, T. A., \& Mulé, N. J. (Eds.). LGBTQ people and social work: Intersectional perspectives. Canadian Scholars' Press, 107-126.

Gouveia, V. V., Milfont, T. L., Fonseca, P. N., \& Coelho, J. A. P. M. (2008). Life satisfaction in Brazil: Testing the Psychometric Properties of the Satisfaction With Life Scale (SWLS) in Five Brazilian Samples. Social Indicators Research, 90(1), 267-277. doi: 10.1007/s11205-008-9257-0

Griep, R. H. ... et al., (2005). Validade de constructo de escala de apoio social do Medical Outcomes Study adaptada para o português no Estudo Pró-Saúde. Cadernos de Saúde Pública, 21(3), 703-714. doi: 10.1590/S0102-311X2005000300004

Herek, G. M. (2002). Heterosexuals' attitudes toward bisexual men and women in the United States. Journal of sex research, 39(4), 264-274.

Kertzner, R. M., Meyer, I. H., Frost, D. M., \& Stirratt, M. J. (2009). Social and psychological well-being in lesbians, gay men, and bisexuals: the effects of race, gender, age, and sexual identity. American Journal of Orthopsychiatry,79(4), 500. doi: 10.1037/ a0016848

Levounis, P., Drescher, J., \& Barber, M. E. (2014). O livro de casos clínicos GLBT. Porto Alegre: Artmed.

Lewis, R. J., Kholodkov, T., \& Derlega, V. J. (2012). Still stressful after all these years: A review of lesbians' and bisexual women's minority stress. Journal of Lesbian Studies, 16(1), 30-44. Doi: 10.1080/10894160.2011.557641

Martell, C. R., Safren, S. A., \& Prince, S. E. (2004). Cognitive-behavioral therapies with lesbian, gay, and bisexual clients. Guilford Press.

Meidlinger, P. C., \& Hope, D. A. (2014). Differentiating disclosure and concealment in measurement of outness for sexual minorities: The Nebraska Outness Scale. Psychology of Sexual Orientation and Gender Diversity, 1(4), 489. doi: 10.1037/sgd0000080

Meyer, I. H. (2003). Prejudice, social stress, and mental health in lesbian, gay, and bisexual populations: Conceptual issues and research 
evidence. Psychological Bulletin, 129(5), 674-697. doi: 10.1037/0033-2909.129.5.674

Meyer, I. H. (2015). Resilience in the study of minority stress and health of sexual and gender minorities. Psychology of Sexual Orientation and Gender Diversity, 2(3), 209. doi: 10.1037/sgd0000132

Muthén, L. K., \& Muthén, B. O. (2010). Mplus: Statistical analysis with latent variables. User's guide. Los Angeles, EUA: Muthén \& Muthén.

Organização Mundal de Saúde (OMS). (1948). International Classification of functioning, disability and health: ICF. Organização Mundial da Saúde.

Pachankis, J. E., Hatzenbuehler, M. L., Rendina, H. J., Safren, S. A., \& Parsons, J. T. (2015). LGB-affirmative cognitive-behavioral therapy for young adult gay and bisexual men: A randomized controlled trial of a transdiagnostic minority stress approach. Journal of Consulting and Clinical Psychology, 83(5), 875-889. doi: 10.1037/ccp0000037

Patton, M. Q. (1990). Qualitative Evaluation and Research Methods. Newbury Park, EUA: Sage Publications, Inc.

Puckett, J. A., Horne, S. G., Herbitter, C., Maroney, M. R., \& Levitt, H. M. (2017). Differences across contexts: Minority stress and interpersonal relationships for lesbian, gay, and bisexual women. Psychology of Women Quarterly, 41(1), 8-19. doi: 10.1177/0361684316655964
Reppold, C. T., Mayer, J. C., Almeida, L. S., \& Hutz, C. S. (2012). Avaliação da resiliência: Controvérsia em torno do uso das escalas. Psicologia: reflexão e crítica, 25(2), 248-255.

Szymanski, D. M., Kashubeck-West, S., \& Meyer, J. (2008). Internalized heterosexism: A historical and theoretical overview. The Counseling Psychologist, 36, 510-524. doi: 10.1177/0011000007309488

Taylor, J. (2018). Bisexual mental health: A call to action. Issues in mental health nursing, 39(1), 83-92. doi: 10.1080/01612840.2017.1391904

Wagnild, G. M. (2010). The Resilience Scale user's guide for the US English version of the Resilience Scale and the 14Item Resilience Scale (RS-14). Worden: The Resilience Center.

Young, J. E., Klosko, J. S., \& Weishaar, M. E. (2008). Terapia do esquema: Guia de técnicas cognitivo-comportamentais inovadoras. Porto Alegre, Brasil: Artmed.

Zimmermann, L., Darnell, D. A., Rhew, I. C., Lee, C. M., \& Kaysen, D. (2015). Resilience in community: A social ecological development model for young adult sexual minority woman. American Journal of Community Psychology, 55(1-2), 179-190. doi: 10.1007/s10464-015-9702-6

Recebido em: 15/05/2018

Reformulado em: 06/02/2019

Aprovado em: 12/04/2019 
Sobre os autores:

Fernanda de Oliveira Paveltchuk é professora substituta do Instituto de Psicologia da Universidade Federal do Rio de Janeiro (UFRJ), psicóloga graduada pela Universidade Federal do Rio de Janeiro (UFRJ) e mestra em Psicologia Clínica pela Pontifícia Universidade Católica do Rio de Janeiro (PUC-Rio). Pesquisa Estresse de Minorias, população LGBT e Terapia Cognitivo-Comportamental.

ORCID: https://orcid.org/0000-0002-8319-874X

E-mail:paveltchuk@gmail.com

Juliane Callegaro Borsa é professora adjunta do Departamento de Psicologia e do Programa de Pós-Graduação em Psicologia Clínica da Pontifícia Universidade Católica do Rio de Janeiro (PUC-Rio), coordenadora do Grupo de Pesquisa em Avaliação Psicológica APlab - Pessoas \& Contextos, coordenadora do Setor de Testes Psicológicos (SETEST PUC-Rio), bolsista produtividade CNPq Nível 2 e bolsista Jovem Cientista do Nosso Estado FAPERJ.

ORCID: https://orcid.org/0000-0001-7703-5509

E-mail: juliborsa@gmail.com

Bruno Figueiredo Damásio é professor do Programa de Pós-Graduação em Psicologia da UFRJ, chefe do Departamento de Psicometria e coordenador do Laboratório de Psicometria e Psicologia Positiva (LP3).

ORCID: https://orcid.org/0000-0002-1150-092X

E-mail: bf.damasio@gmail.com

Contato com os autores:

Av. Pasteur, 250, Urca

Rio de Janeiro-RJ

CEP: 22290-250 